\title{
Lactam Amide Spinning
}

\author{
Tomohiko Ohwada* and Yuko Otani \\ * Graduate School of Pharmaceutical Sciences, The University of Tokyo \\ 7-3-1 Hongo, Bunkyo-ku, Tokyo 113-0033, Japan
}

(Received August 21, 2020; E-mail: ohwada@mol.f.u-tokyo.ac.jp, otani@mol.f.u-tokyo.ac.jp)

\begin{abstract}
The amide rotation of cis-trans lactams is fundamentally important, but has rarely been studied, with the exception of reports on peptide-based lactams. Here, we find a consistent relationship between the trans/cis ratio of the lactam amide and the trans/cis-amide rotation rate upon elongation of the stapling side chain of two 7-azabicyclo[2.2.1] heptane bicyclic units linked via a non-planar amide bond. That is, as the chain length increases, the spinning rate from trans-to cis-lactam amide decreases, resulting in an increase in the trans ratio. This chain length dependence of lactam amide isomerization and our simulation studies support the idea that the current lactam amide can rotate 360 degrees due to the occurrence of nitrogen pyramidalization of lactam. The direction of tilting of the pyramidalization of nitrogen atoms in the bicyclic system is synchronous with the direction of the semicircular rotation of the amide.
\end{abstract}

\section{Introduction}

As described in more detail in biochemistry textbooks than in organic chemistry textbooks, the amide functional group takes a planar structure due to the $\mathrm{C}=\mathrm{N}$ double bonded resonance structure. There is therefore a rotational barrier with respect to the amide plane, which is important for the structuring of proteins and peptides. Amide rotation of lactams (cyclic amides) is also an important aspect for the accessibility and limitation of lactam conformations such as cyclic peptides and lactam compounds. Many bioactive cyclic peptides and lactams, both natural and non-natural, have been reported and have received a great deal of attention. However, the chemistry of lactam amide rotation has been little studied. Amide rotation is of fundamental importance as it is involved in a wide range of structural phenomena, from protein folding ${ }^{1-4}$ to conformational multiplicity of cyclic peptides and lactam compounds. $^{5-10}$

\section{Lactam Amide Spinning}

\subsection{Analysis of Spinning of Planar Amide Lactams}

In the case of lactams, the rotation of the amide is affected by the length of the intervening chain and the nature of the rotatable bond. If the amide bonds of the lactam are able to spin freely through 360 degrees, as in the case of open chain amides, then a simple correlation should be observed between the trans/cis isomer ratio of the amide and the rate of rotation between the trans and cis amide conformers, e.g., as the rate of rotation from the trans isomer to the cis isomer increases, the ratio of cis-isomers should increase. That is, if the amide bond is monotonically rotated 360 degrees, the conservation of angular momentum (I), i.e., the relationship $\omega=\mathbf{I} /\left(\boldsymbol{m} \boldsymbol{r}^{2}\right)$, where $\omega, \boldsymbol{r}$, and $\mathbf{m}$ are the angular velocity, radius and molecular weight, respectively. That is, the longer the chain length $(2 \pi r$, which is approximately equal to the perimeter of the cyclic structure) (or the greater the value of $\boldsymbol{r}$ retrospectively), the smaller the rotational velocity $\boldsymbol{k}(\omega)$ (Figure 1$)$.

However, it is well known that the rotation of lactam amides is restricted ${ }^{11-14}$ and that the amide bonds of lactam cannot spin through 360 degrees due to ring coiling

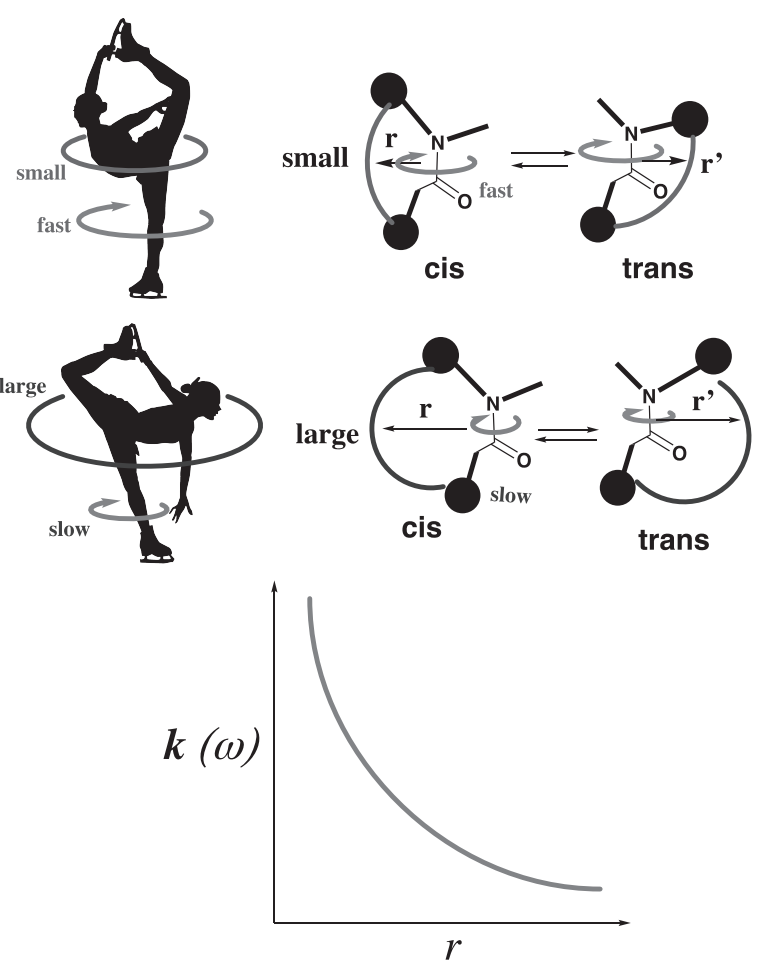

Figure 1. A simple correlation between the chain length (approximately $2 \pi \boldsymbol{r}$ ) and rotational velocity $\boldsymbol{k}(\omega)$, supposing the amide bond is monotonically rotated 360 degrees like a skater.

(Figure 2(1)). This always occurs independently of the size of the ring.

For example, a simple $N$-methyl tertiary amide lactam, $1-$ methylarazacycloundecan-2-one L11 (11-membered ring), with a medium-membered ring size, showed a discontinuous rotation of the lactam amide bond and did not rotate 360 degrees. An energy landscape of 1-methylazacycloundecan-2one $\mathbf{L 1 1}$ with respect to two dihedral angles, amide rotation $\omega$, and a puckering angle $(\mathrm{C}-\mathrm{C}-\mathrm{N}-\mathrm{C}(=\mathrm{O}), \phi)$, obtained in the metadynamics calculations in the presence of explicit solvent water molecules (Figure 3), indicated that there are two con- 
(1) Planar amide lactam

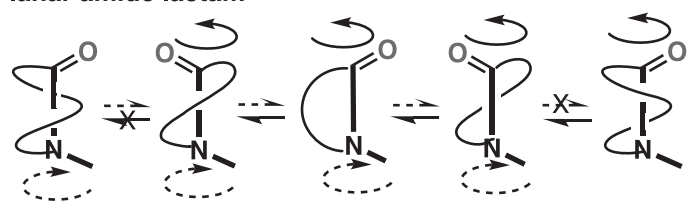

(2) N-Pyramidal amide lactam

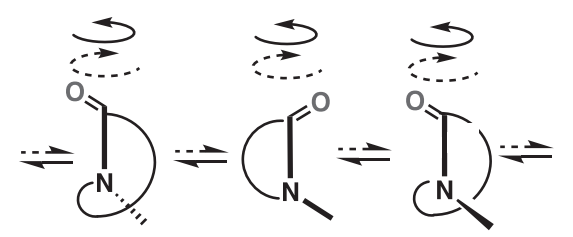

Figure 2. (1) Hindered rotation of coiled planar lactam amides (2) 360 -degree rotation of an $N$-pyramidalized lactam.

formations arising from ring puckering, and in both cases, there is a high energy barriers (white-colored regions) along the rotation of amide bond ( $\omega$ : from $-180^{\circ}$ (trans) to $+180^{\circ}$ (trans) through $0^{\circ}(\mathrm{cis})$ ). This strongly indicated that the lactam amide cannot spin through 360 degrees.
L11

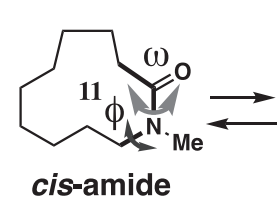

$\omega=0^{\circ}$

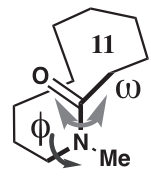

trans-amide

$\omega=+180^{\circ}$

$-180^{\circ}$

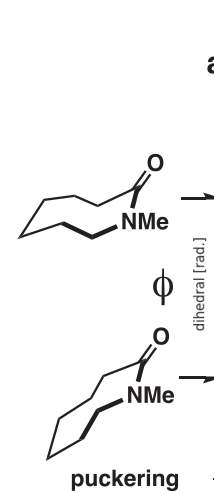

puckering

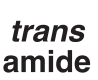

amide trans

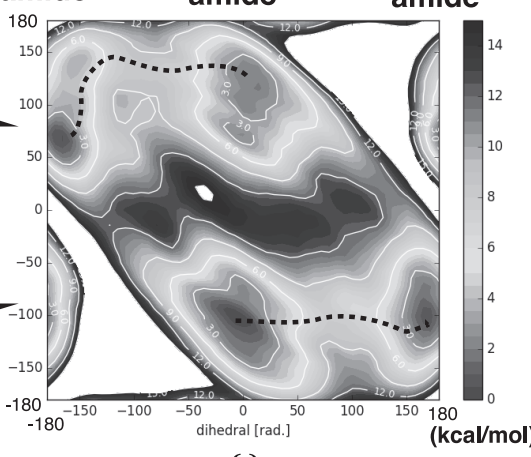

$\omega$

amide rotation

Figure 3. Energy landscape $(\Delta G(300 \mathrm{~K}))$ for rotation of planar lactam amide of 1-methylarazacycloundecan-2-one L11. White region is higher than $15 \mathrm{kcal} / \mathrm{mol}$.

Several studies have characterized spinning of lactam amides. Indolactam V (Figure 4), isolated from actinomycetes, is a minimal pharmacophore for the tumor-promoting activity of teleocidin, which binds and activates various kinase receptors, including protein kinase C. Due to its potent activity and unique nine-membered lactam structure, teleocidin derivatives have been studied in medicinal chemistry and organic chemistry. Endo et al. showed that indolactam V takes several distinct conformations in equilibrium. ${ }^{15}$ High-temperature straightforward molecular dynamics (MD) calculations of derivatives of indolactam $\mathrm{V}$ suggested that the nine-membered lactam moiety takes 10 conformations, but only two structures derived from cis-lactam amide (TWIST) and trans-lactam amide (SOFA) are stable in indolactam V. ${ }^{15}$ Recently, the Abe's group found that thioindolactam V (Figure 4), a derivative of indolactam $\mathrm{V}$ produced by the single-atom substitution of $\mathrm{NMe}$ to sulfur through the action of artificial biosynthetic enzymes, has a completely different conformational selectivity, and the calculations suggested the presence of multiple conformations of thioindolactam $\mathrm{V}$ in a similar manner of indolactam V. However, the high energy barriers may not allow these conformations to be interconverted, indicating that each conformation is rather isolated. ${ }^{16}$ These examples are consistent with the fact that lactam amides cannot rotate 360 degrees, and spinning features is dramatically changed by even a single atom replacement.

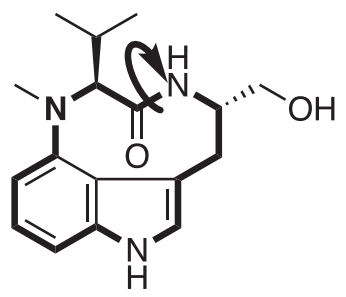

Indolactam V

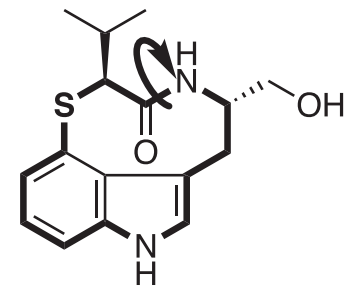

\section{Thioindolactam V}

Figure 4. Other examples of lactam amide rotation, defining conformational distribution.

The rotation rate of lactam amides has rarely been studied, but it has been reported by Shi and Rabenstein ${ }^{11-13}$ that the rotation rates of $\alpha$-proline amides $(\mathbf{S 1}-\mathbf{S 4})$ associated with intramolecular disulfide bond formation is not consistently dependent on the cis-trans isomer ratio and the chain length dependence of the cis-trans interconversion rate. They changed the number of intervening amino acids, which corresponds to the change of ring size of $\alpha$-proline amide lactam (S1-S4). The cis-trans isomer ratio did not show consistent change upon the increase of the number of the intervening amino acid residues (that is, lactam ring size). While the interconversion rate from cis to trans $\left(k_{\mathrm{c} \rightarrow \mathrm{t}}\right)$ decreased as the lactam ring size increased, the alternative interconversion rate from trans to cis $\left(k_{\mathrm{t} \rightarrow \mathrm{c}}\right)$ changed randomly (Figure 5).
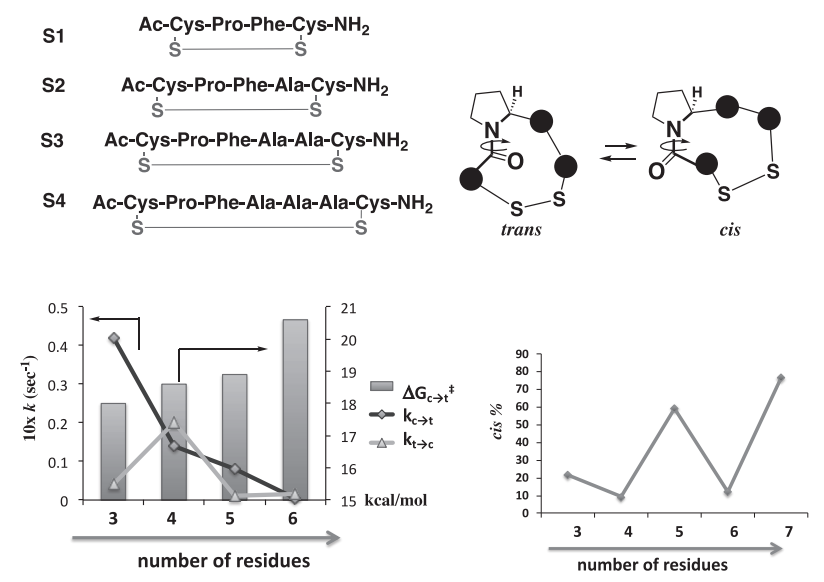

Figure 5. Rabenstein's studies of rotation rate of $\alpha$-proline amides associated with intramolecular disulfide bond formation.

This may be due to the discontinuous rotation of the amide bond and the relatively high ( $\sim 20 \mathrm{kcal} / \mathrm{mol})$ energy barrier for amide rotation. In fact, our metadynamics simulations of $\mathbf{S 4}$ gave the energy landscape with respect to two dihedral angles, 
amide rotation angle $(\omega, \mathrm{C} 1-\mathrm{N} 2-\mathrm{C} 3-\mathrm{C} 4)$ and disulfide bond rotation, $\mathrm{C}_{1^{\prime}}-\mathrm{S}_{2},-\mathrm{S}_{3^{\prime}}-\mathrm{C}_{4^{\prime}}(\phi)$ (Figure 6). This map indicated that rotation of proline amide from trans conformation (A) to the cis conformation (B) is marginally possible, but reaching to trans conformation (A') will be hampered by high activation energy. Thus, this observation suggests that lactam amide rotation is limited and that the general trend is that the inability of lactam amide bonds to spin through 360 degrees is probably due to ring coiling (Figure 2(1)).
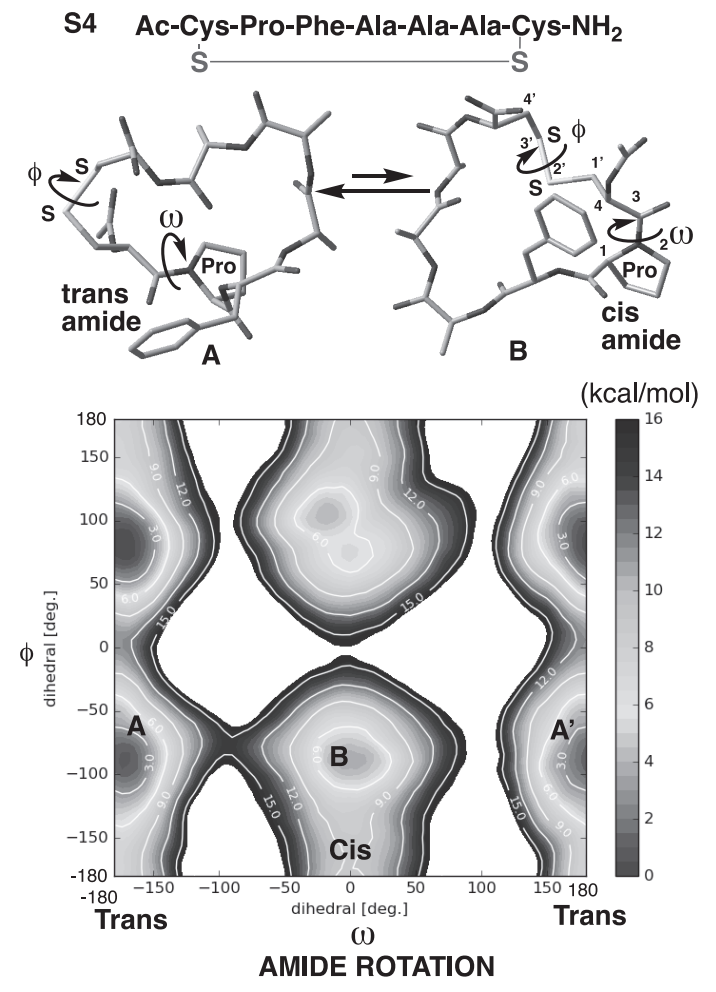

Figure 6. Discontinuous amide rotation of disulfide $\alpha$-proline lactam (S4). Energy landscape $(\Delta G(300 \mathrm{~K}))$. White region is higher than $15 \mathrm{kcal} / \mathrm{mol}$.

\section{Spinning of $N$-Pyramidalized Amide Lactams}

\subsection{Nitrogen Pyramidal Amide Unit and Design of Lactams}

Although non-planar amide systems have been extensively studied, ${ }^{17-25}$ their characteristic structural features and the associated reduction of the amide rotational barrier have rarely been used in molecular design, mainly due to the instability of the amide bonds under conventional conversion conditions. However, an examination of the tertiary amides of 7azabicyclo[2.2.1.1] heptane has shown that this bicyclic system induces nitrogen pyramidation (Figure 7), ${ }^{26}$ and these amides have been shown to be sufficiently stable to undergo further conversions. $^{27}$

The rotational barrier for amide cis-trans isomerization of nitrogen pyramidal amides decreases in solution, indicating easier amide rotation. The inversion of the nitrogen atoms of the amides is a low energy process (calculated activation energy: less than $3 \mathrm{kcal} / \mathrm{mol}$ ) (Figure 7) and nitrogen pyramiding is also an easy process. Furthermore, derivatives with carboxylic acid functionality (Ah2c, Figure 8) can be regarded as conformationally constrained $\beta$-proline surrogates; for $7-$ azabicyclo[2.2.1]heptan-2-carboxylic acid with an alkoxymethyl substituent at the $\mathrm{C} 4$-bridgehead position, the presence

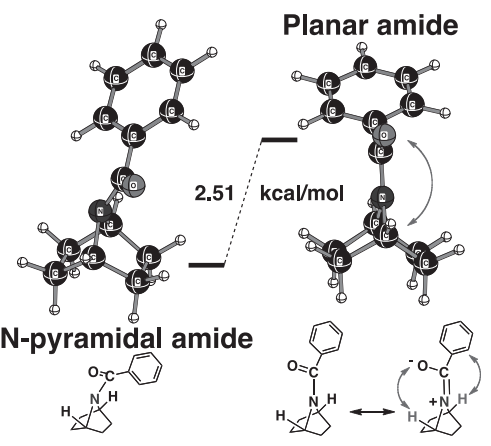

Figure 7. Nitrogen pyramidalization of 7-azabicyclo[2.2.1]heptane amide.

of a bridgehead substituent is regardless of the solvent, the cis-trans equilibrium of the amide is completely biased to one

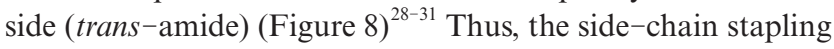
of the bicyclic amide dimer is a very clear way to understand the thermodynamics and kinetics of non-planar amide lactam rotation.
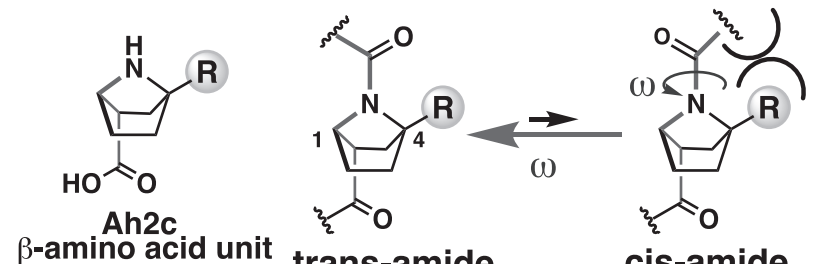

cis-amide

Figure 8. A single substitution can control amide cis-trans nitrogen pyramidalization of 7-azabicyclo[2.2.1] heptane amide.

Therefore, we expected that this bicyclic system would provide a suitable scaffold on which to study the lactam-amide ratio and the dependence of rotation on staple length. That is, as the side chain staple length decreases in the bicyclic dimer (Figure 9), the trans-to-cis rotation of the amide consistently decelerates, while at the same time the cis-to-trans rotation of the amide accelerates, ultimately resulting in a very large dynamic range of variation in the cis-trans ratio of the amide, depending on the side chain staple length. We found a simple relationship in which the cis-trans equilibrium of the amide is extended until it is completely inverted. This is consistent with the idea that the current bicyclic amide lactams can rotate 360 degrees with respect to the amide bond due to the pyramidalization of the amide nitrogen atom (Figure 2(2)).

\subsection{Synthesis of Bicyclic Dimer Lactams}

We used ring-closing metathesis (RCM) to staple model bridged $-\beta$-proline dimers with alkenyl side chains of various lengths (Figure 9). Ring-closing dimeric amides with different

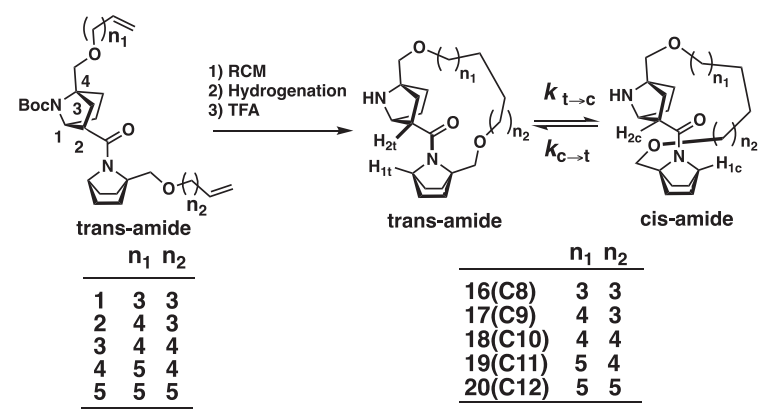

Figure 9. Bicyclic dimer lactams. 
length of linkers (16-20) were synthesized as shown in Figure 10. RCM reactions of $\mathbf{1 - 5}$, which are Boc-protected open-chain model dimers with alkenyl side chains of various lengths, were performed using Grubbs I catalysts to obtain products 6-10 stapled as mixtures of $\mathrm{E}$ and Z-olefins in good yields. Hydrogenation of alkenes yielded saturated compounds 11-15, respectively; treatment of 11-15 with TFA removed the Boc group, giving dimeric amines (16(C8), 17(C9), 18(C10), 19(C11) and 20(C12)) (Figure 10).
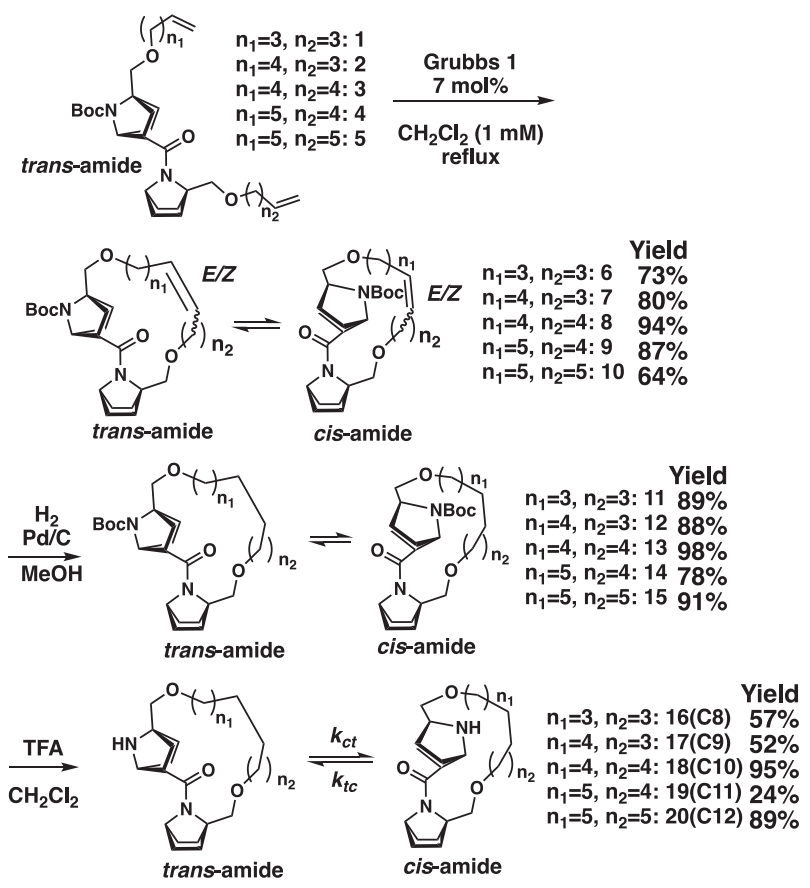

Figure 10. Synthesis of stapled $N$-pyramidalized amide dimer lactams.

\subsection{Spinning Features of Bicyclic Dimer Lactams}

Amide cis-trans Isomerization From the NMR spectra in $\mathrm{CD}_{2} \mathrm{Cl}_{2}$, the cis/trans ratio of the amide of the dimer $(\mathbf{1 6}(\mathbf{C 8})-$ 20(C12)) was estimated. ${ }^{32}$ It was found that the ratio varied significantly with the length of the linker (Figure 11). As the linker length increases, the ratio of $c i s$-amides increases, changing from almost exclusively trans $(\mathbf{6}(\boldsymbol{Z})-\mathbf{N H}(\mathbf{C 8}), 99.9 \%$ trans, $\left.\mathrm{K}_{\mathrm{t} / \mathrm{c}}>999\right)$ to predominantly cis (20(C12), 94\% cis,

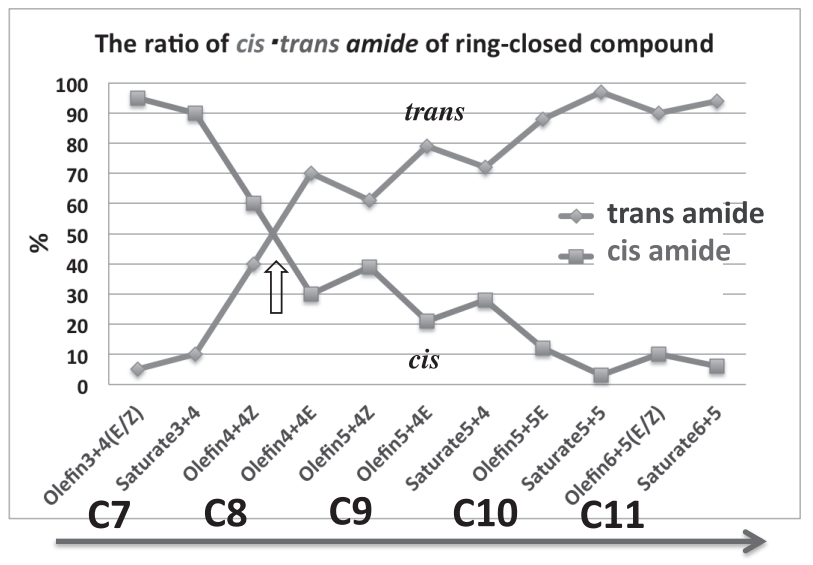

Linker length increases

Figure 11. Equilibrium constants of amide cis-trans isomerization of $N$-pyramidalized amide dimer lactams.
$\left.\mathrm{K}_{\mathrm{t} / \mathrm{c}}=0.06\right)$. There is also a clear difference in cis or trans amide selectivity between $\mathbf{1 7 ( C 9 )}$ and 18(C10). We found that $\mathbf{1 6 ( C 8 )}$ and 18(C10) exhibit similar trans/cis ratios in $\mathrm{CD}_{2} \mathrm{Cl}_{2}$ (16: 8/92; 18: 73/27) and protic $\mathrm{CD}_{3} \mathrm{OD}(16: 19 / 81 ; 18: 77 / 23)$, showing independency of solvent polarity. In fact, $\mathbf{1 6}(\mathbf{C 8})$ is the first example of a bridgehead-substituted bicyclic system that takes the reverse (cis) amide structure. Given that the stapling rings of these compounds are of medium size (18 to 22 members), these results are unprecedented.

\subsection{Chain-length-dependent Amide Rotation Kinetics}

The rotational kinetics of amide bonds of bicyclic dimers with different linker lengths (16(C8), 17(C9), 18(C10) and 19(C11)) were estimated using exchange spectroscopy (EXSY).

The kinetics of 17(C9), 18(C10), and 19(C11) were measured in $\mathrm{CDCl}_{3}$, and the kinetics of 16(C8), 17(C9), and 18(C10) were measured in $\mathrm{CD}_{3} \mathrm{OD}$ (Figure 12). The rates of exchange from trans to cis $\left(k_{t \rightarrow c}\right)$ and cis to trans $\left(k_{c \rightarrow t}\right)$ at each temperature were obtained by fitting to a modified version of the reported theoretical equation: the rotation rate in $\mathrm{CD}_{3} \mathrm{OD}$ is clearly smaller than that in $\mathrm{CDCl}_{3}$, which means that the rotational process was suggested that the solvation effect, such as entropy, polarity and viscosity of the solvent, may contribute to this motion. The obtained trans to cis exchange rate
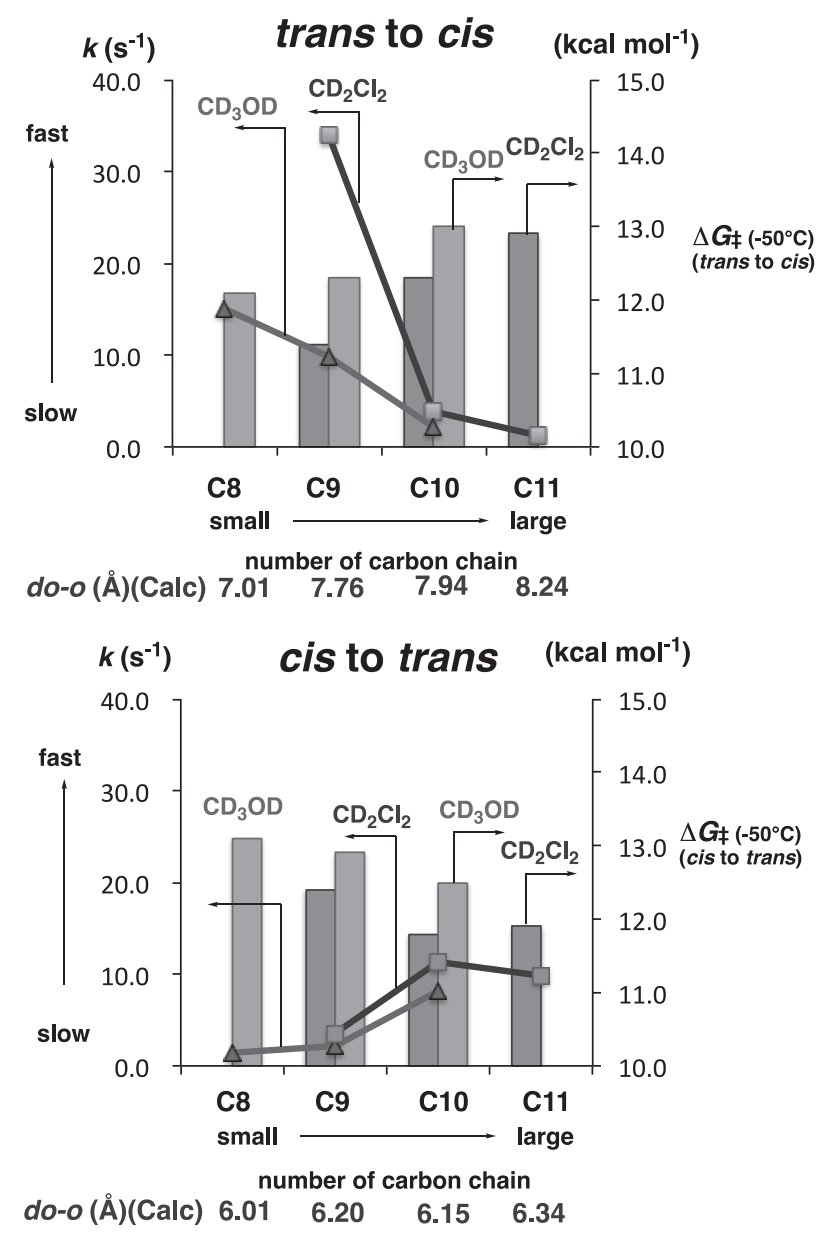

Figure 12. (a) Staple length dependence of the isomerization rate of the trans-cis-amide-isomer. (b) Dependence of the isomerization rate of the cis-trans amide isomer on the number of chain carbon atoms. Left axis: upper line: spinning rate at $\mathrm{CD}_{2} \mathrm{Cl}_{2}\left(-50{ }^{\circ} \mathrm{C}\right)$; lower line: turnover rate at $\mathrm{CD}_{3} \mathrm{OD}\left(-43^{\circ} \mathrm{C}\right)$. Right axis: rotational barrier at $\mathrm{CD}_{2} \mathrm{Cl}_{2}\left(\Delta G^{\ddagger}\left(-50.0^{\circ} \mathrm{C}\right)\right)$, red line: rotational barrier at $\mathrm{CD}_{3} \mathrm{OD}\left(-50.0^{\circ} \mathrm{C}\right)$. 
$\left(k_{t \rightarrow c}\right)$ increased dramatically with decreasing ring size (28-fold for $\left.\mathrm{CDCl}_{3}\right)($ Figure 12), whereas the cis to trans exchange rate $\left(k_{t \rightarrow c}\right)$ increased slightly with increasing linker size (Figure 12), which is supportive for an idea that the cis/trans ratio and the spinning rate of lactam amide are consistent.

\subsection{Energy Landscape of the Lactam Amide Rotation}

Metadynamics simulations of bicyclic amides 17(C9) and 19(C11) (Figure 13(a,b)) revealed that this system is an example of a 360-degree rotation of lactam amide bond due to nitrogen pyramidalization: in principle, the rotation of the lactam amide (dihedral angle $\mathrm{C}($ bridgehead) $\mathrm{NC}(\mathrm{O}) \mathrm{C}$ ) is restricted to a south semicircle-rotation angle (e.g., $\omega: 0^{\circ}$ to $+180^{\circ}$ ) or a north semicircle-rotation angle (e.g., $\omega$ : $-180^{\circ}$ to $0^{\circ}$ ) with respect to the amide dihedral angle $(\omega)$ due to the ring coiling (see Figure 2 (1)), but in the present case (17(C9) and 19(C11)), lactam amide rotation can undergo continuous north semicircle-rotation with inward-tilted amide nitrogen pyramidalization (dihedral angle $\phi=150^{\circ}$ in Figure 13(a, b), B to A)) and at the same time continuous south semicircle-rotation with outward-tilted amide nitrogen pyramidalization (dihedral angle $\phi=100^{\circ}$ in Figure 13(a, b), A to B'). The rotation is bi-directional. The difference between the energy barrier ( $\sim 4 \mathrm{kcal} / \mathrm{mol}$ at $b)$ in north semicircle-rotation $(\mathrm{a} \rightarrow \mathrm{d}$, Figure 13(a): trans $\rightarrow$ cis $)$ and the energy barrier $(\sim 7 \mathrm{kcal} / \mathrm{mol}$ at e) in south semicircle-rotation ( $\mathrm{d} \rightarrow \mathrm{e}$, Figure 13(a): cis $\rightarrow$ trans) is consistent with the difference in observed rotation speed $\left(k_{t \rightarrow c}>\right.$ $k_{c \rightarrow t}$ ) of 17(C9) (Figure 12). In contrast, the trans amide of the $N$-tosylated derivative of $\mathbf{8}(E)(\mathbf{T s}-\mathbf{8}(E)(C 10))$ takes a pyramidalized structure with the nitrogen of the inwardly tilted amide in the crystal state (Figure 14). The DFT-calculated cis-amide of $16(\mathrm{C} 8)-19(\mathrm{C} 11)$ also shows the pyramidalization of outwardly tilted amide nitrogen, and the DFT-calculated trans amide of 16(C8)-19(C11) also shows the pyramidalization of inwardly tilted amide nitrogen (Figure 14). In conventional amide lactams, the ring coiling restricts the amide dihedral angle to a southern semicircular rotation angle (e.g., $0^{\circ}$ to $+180^{\circ}$ ) or a northern semicircular rotation angle (e.g., $-180^{\circ}$ to $0^{\circ}$ ) with respect to the amide dihedral angle $(\omega)$ (see Figure 2(1)), $\omega-180^{\circ}$ to $+0^{\circ}$ ).

On the other hand, metadynamics simulations of hypothetical monocyclic pyrrolidine amides (21(S-C9), 22(S-C11), Figure 13(c) and (d)) in which 7-azabicyclo[2.2.1.1]heptane is replaced by a monocyclic pyrrolidine derivative $(21(S-C 9)$, 22(S-C11), Figure 13(c) and (d)), show that one dihedral angle around the amide nitrogen atom is allowed and the amide rotation relative to coupling has been shown to be limited to the southern semicircular rotation angle (e.g., $0^{\circ}$ to $+180^{\circ}$, Figure 13(c, d), A to B): a north semicircle-rotation (e.g., angle $\omega:-180^{\circ}$ to $0^{\circ}$, Figure $13(\mathrm{c}, \mathrm{d}), \mathrm{B}^{\prime}$ to A) is inhibited by the high activation barrier. Since monocyclic pyrrolidine amide takes a planar amide structure, nitrogen pyramiding is important for 360-degree rotation of the lactam amide. A similar rotational limitation was observed when the lower bicyclic amide was replaced by a simple acyclic $\mathrm{N}$-methylamide (Figure 13(e) and 22(C11-N-Me-amide)), probably due to the planar amide.

3.6 Synchronized Tilting Direction of the Pyramidal Nitrogen Atom with Lactam Rotation

The crystal structures of the derivatives (6-20) suggest that the amides in the ground state (trans and cis isomers) are tilted in the opposite direction to the bridgehead substituents (Figure 14(b)). All the DFT-computed structures of lactam
16(C8)-19(C11) are consistent with this idea that the amide (trans and cis isomers) in the ground state is tilted in the opposite direction to the bridgehead substituent (Figure 14). Tilting of the amide on the same side as the bridgehead substituent induced significant destabilization (calculated as $>60 \mathrm{kcal} /$ mol). The tilte angle $(\alpha)$ was $25.0^{\circ}$ in the trans-state and $20.7^{\circ}$ in the $c i s^{-}$state (Figure 14(a)). These structural features are the result of the easy nitrogen pyramiding of the bicyclic lactam amide.

\section{Conclusion}

The side chain stapling of these bicyclic dimeric amides (lactam) showed a monotonic relationship between the spinning rates of cis and trans lactam amides and the ratio of trans/ cis isomers, depending on the size of the ring: as the ring size of the stapling structure is increased, the spinning rate from trans to cis decreases and that from cis to trans increases, resulting in an increase in the trans/cis ratio. This results in a complete reversal of the amide equilibrium and an extreme range of trans/cis ratios $(\sim 0 / 1-1 / 0)$ depending on the length of the linker is observed. Our observations and simulation results suggest that the current lactam amide can be rotated 360 degrees due to the occurrence of nitrogen pyramidalization. The direction of tilting of the pyramidalization of nitrogen atoms in the bicyclic system is synchronized with the direction of the semicircular rotation of the amide. Our results provide new insights into the amide rotation of lactams, a ubiquitous and divergent class of compounds. The rotational properties of the lactam amides observed here are generalized and have potential applications in molecular design.

\section{(a) 17(C9)}

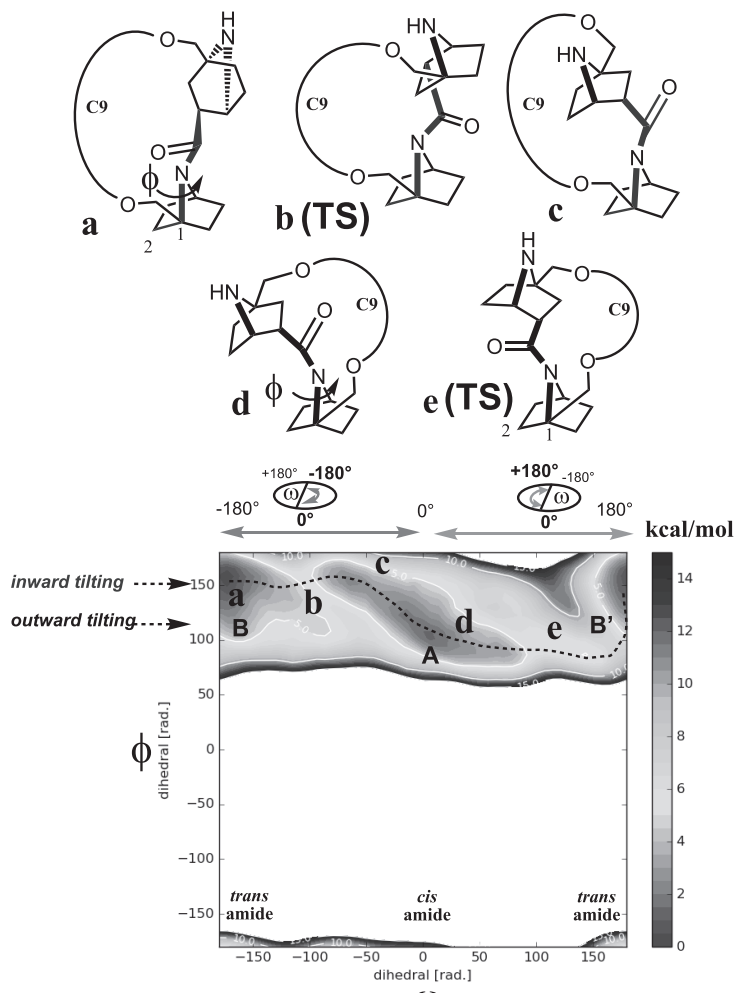

$\omega$ 
(b) $19(\mathrm{C} 11)$

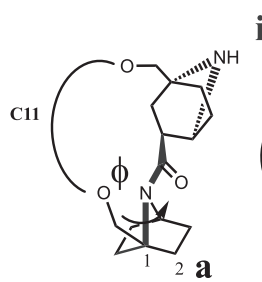

inward tilting

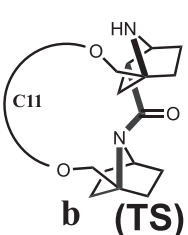
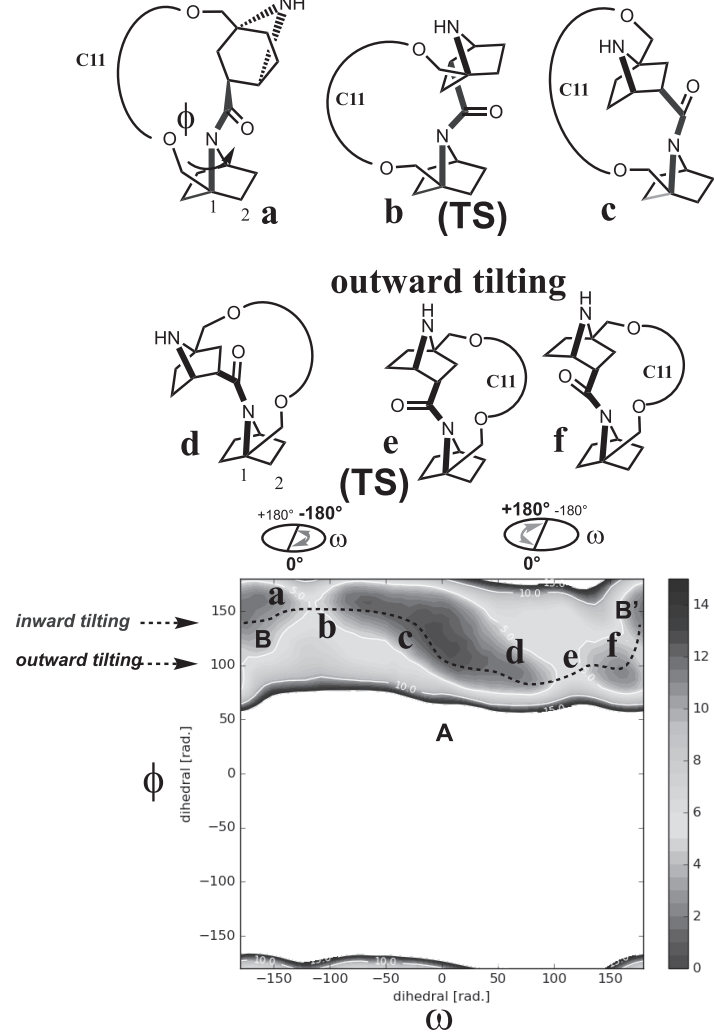

(c) $21(\mathrm{~S}-\mathrm{C} 9)$

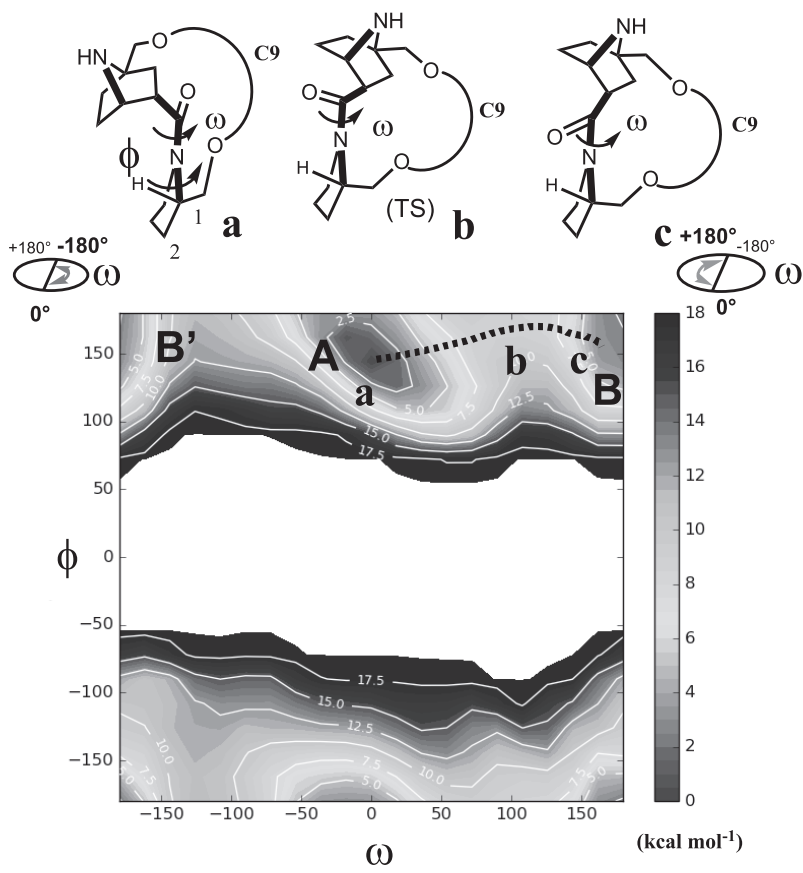

(d) 22(S-C11)

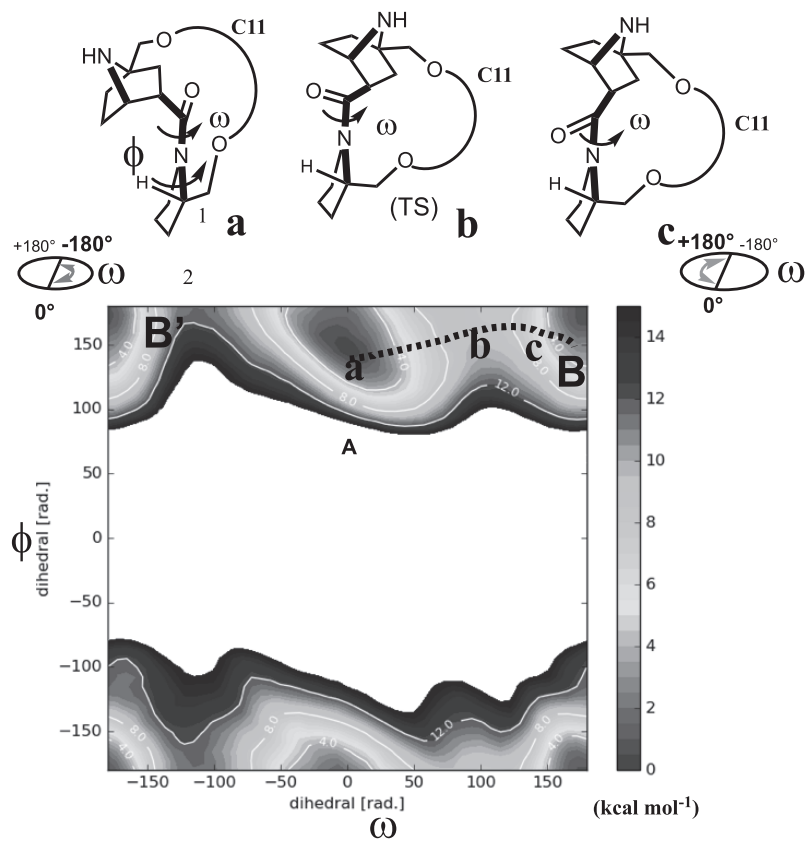

(e) 22(C11-N-Me-amide)

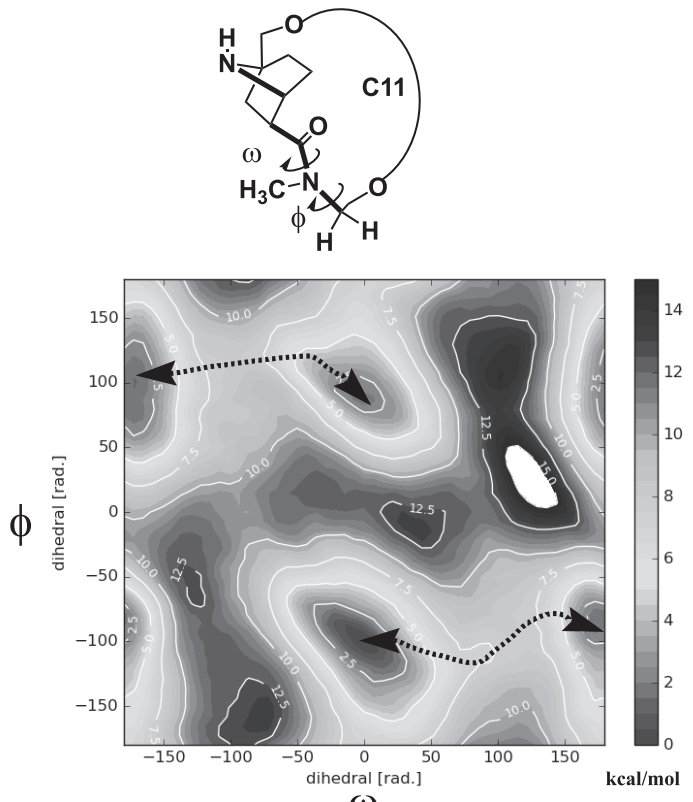

$\omega$

Figure 13. Metadymamic simulations of the landscape of bicyclic and monocyclic lactam amide rotation. (a) Bicycle lactam 17(C9). (b) Bicycle lactam 19(C11). (c) Monocycle lactam 21(S-C9). (d) Monocycle lactam 22(S-C11). (e) Bicyclic and $\alpha$-amino acid combination C11-N-Me-amide. Dashed lines indicate the rotational pathways. Energy Landscape $(\Delta G(300 \mathrm{~K})$ ). White region is higher than $15 \mathrm{kcal} / \mathrm{mol}$.

\section{Acknowledgements}

We thank our collaborators, Xin Lu (Takeda), Hisashi Ohno, Dr. Luhan Zhai, and Dr. Siyuan Wang, Dr. Aoze Su (Astellas) and Dr. Seokhwi Park. We also thank Prof. Kentaro
Yamaguchi and Dr. Masatoshi Kawahata, Tokushima Bunri University, Kagawa Campus, Faculty of Pharmaceutical Sciences for their continuous contributions to crystal structure studies. The computations were performed at the Research 
(a)
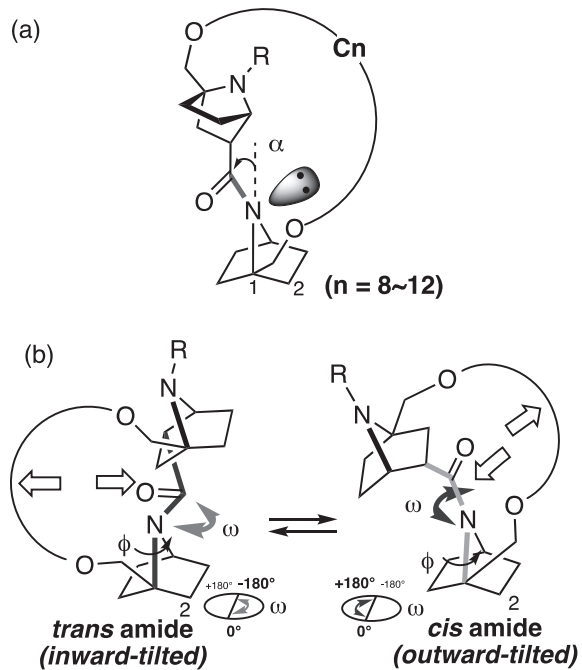

$\omega=\angle \mathrm{C}($ bridgehead $) \mathrm{NC}(\mathrm{O}) \mathrm{C}$

$\phi=\angle \mathrm{CC}($ bridgehead $) \mathrm{NC}(\mathrm{O})$

Figure 14. (a) Nitrogen pyramidalization (b) Equilibrium of trans amides and cis amides in bicyclic lactams. (3) 360-degree rotation of an $\mathrm{N}$-pyramidalized lactam.

Center for Computational Science, Okazaki, Japan. Some computations were performed as part of the "High Performance Computing Infrastructure (HPCI)” project (hp150177 and hp170146), and some were done on the TSUBAME2.5 and TSUBAME3.0 supercomputers at Tokyo Institute of Technology as a part of the TSUBAME encouragement program for female users (16IJ0003). This work was partially supported by JSPS KAKENHI Grant Numbers JP 26104508 and 16K08157 (YO) and 26293002 (TO).

\section{References}

1) Torbeev, V. Y.; Hilvert, D. B. Proc. Natl. Acad. Sci. 2013, 110, 20051.

2) Reimer, U.; Fischer, G. Biophys. Chem. 2002, 96, 203.

3) Lu, K. P.; Finn, G.; Lee, T. H.; Nicholson, L. K. Nat. Chem. Biol. $\mathbf{2 0 0 7 , 3 , 6 1 9 .}$

4) Lummis, S. C. R.; Beene, D. L.; Lee, L. W.; Lester, H. A.; Broadhurst, W.; Dougherty, D. A. Nature 2005, 438, 248.

5) Creighton, C. J.; Reynolds, C. H.; Lee, D. H. S.; Leo, G. C.; Reitz, A. B. J. Am. Chem. Soc. 2001, 123, 12664

6) Kern, D.; Schutkowski, M.; Drakenberg, T. J. Am. Chem. Soc. 1997, $119,8403$.

7) Malešević, M.; Schumann, M.; Jahreis, G.; Fischer, G.; Lücke, C. ChemBioChem 2012, 13, 2122.

8) Hill, T. A.; Shepherd, N. E.; Diness, F.; Fairlie, D. P. Angew. Chem. Int. Ed. 2014, 53, 13020.

9) Kaul, R.; Surprenant, S.; Lubell, W. D. J. Org. Chem. 2005, 70, 3838.

10) Dugave, C.; Demange, L. Chem. Rev. 2003, 103, 2475.

11) Rabenstein, D. L.; Shi, T.; Spain, S. J. Am. Chem. Soc. 2000, 122, 2401.

12) Shi, T.; Rabenstein, D. L. J. Am. Chem. Soc. 2004, 126, 790.

13) Shi, T.; Spain, S. M.; Rabenstein, D. L. Angew. Chem. Int. Ed. 2006, 45,1780

14) Yamada, S.; Homma, A. Chem. Commun. 2002, 334, 2656.

15) Endo, Y.; Ohno, M.; Hirano, M.; Itai, A.; Shudo, K. J. Am. Chem. Soc. 1996, 118. 1841.

16) Morita, I.; Mori, T.; Mitsuhashi, T.; Hoshino, S.; Taniguchi, Y.; Kikuchi, T.; Nagae, K.; Nasu, N.; Fujita, M.; Ohwada, T.; Abe, I. Angew. Chem. Int. Ed. 2020, 59, 3988.

17) Wang, Q. P.; Bennet, A. J.; Brown, R. S.; Santarsiero, B. D. J. Am. Chem. Soc. 1991, 113, 5757

18) Yamada, S. Angew. Chem., Int. Ed. Engl. 1993, 32, 1083.

19) Komarov, I. V.; Yanik, S.; Ishchenko, A. Y.; Davies, J. E.; Goodman, J. M.; Kirby, A. J. Am. Chem. Soc. 2015, 137, 926.

20) Morgan, J.; Greenberg, A. J. Phys. Org. Chem. 2012, 25, 1422.

21) Tani, K.; Stoltz, B. M. Nature 2006, 441, 731.
22) Liniger, M.; VanderVelde, D. G.; Takase, M. K.; Shahgholi, M.; Stoltz, B. M. J. Am. Chem. Soc. 2016, 138, 969.

23) Szostak, M.; Yao, L.; Day, V. W.; Powell, D. R.; Aubé, J. J. Am. Chem. Soc. 2010, 132, 8836

24) Hutchby, M.; Houlden, C. E.; Haddow, M. F.; Tyler, S. N. G.; LloydJones, G. C.; Booler-Milburn, K. I. Angew. Chem. Int. Ed. 2012, 51, 548

25) Pace, V.; Holzer, W.; Meng, G.; Shi, S.; Lalancette, R.; Szostak, R.; Szostak, M. Chem. Eur. J. 2016, 22, 14494.

26) Otani, Y.; Nagae, O.; Naruse, Y.; Inagaki, S.; Ohno, M.; Yamaguchi, K.; Yamamoto, G.; Uchiyama, M.; Ohwada, T. J. Am. Chem. Soc. 2003, 125, 15191.

27) Ocampo Gutiérrez de Velasco, D.; Su, A.; Zhai, L.; Kinoshita, S.; Otani, Y.; Ohwada, T. Molecules 2018, 23, 2363.

28) Wang, S.; Taniguchi, T.; Monde, K.; Kawahata, M.; Yamaguchim, K.; Otani, Y.; Ohwada, T. Chem. Commun. 2016, 52, 4018.

29) Hosoya, M.; Otani, Y.; Kawahata, M.; Yamaguchi, K.; Ohwada, T. J. Am. Chem. Soc. 2010, 132, 14780. Originally the definition of cis and trans amides was reverse in this paper. See Figure 8.

30) Wang, S.; Otani, Y.; Liu, X.; Kahagata, M.; Yanaguchi, K.; Ohwada, T. J. Org. Chem. 2014, 79, 5287.

31) Zhai, L.; Wanh, S.; Nara, M.; Takeuchi, K.; Shimada, I.; Otani, Y.; Ohwada, T. J. Org. Chem. 2018, 83, 13063,

32) Otani, Y.; Liu, X.; Ohno, H.; Wang, S.; Zhai, L.; Su, A.; Kawahata, M.; Yamaguchi, K.; Ohwada, T. Nature Commun. 2019, 19, 461.

PROFILE

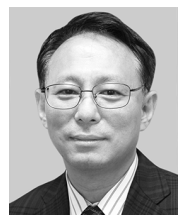

Tomohiko Ohwada received his B.S. and Ph.D. from Graduate School of Pharmaceutical Sciences, The University of Tokyo, Tokyo, Japan, in 1982 and in 1987 under the guidance of Prof. Koichi Shudo. He became an assistant professor of the Shudo group in 1985 before his Ph.D. He made a short visit study at University of Alabama at Birmingham, Department of Chemistry, U.S.A. to relearn computational chemistry from Prof. Koop Lammertsma (now Professor Emeritus, Vrije Universiteit Amsterdam, Netherland). He became a full professor at Faculty of Pharmaceutical Sciences, Nagoya City University, Nagoya, Japan, in 1998, and he was appointed to a professor of Graduate School of Pharmaceutical Sciences, The University of Tokyo, in 2001. His research interest includes structural chemistry of peculiar molecules such as nonplanar amides, cation chemistry and medicinal chemistry. He has been trying to combine calculations with experiments since his graduate student day, for more than 35 years.

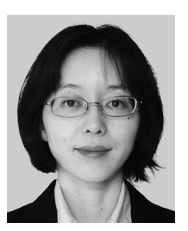

Yuko Otani received her Ph.D. from Graduate School of Pharmaceutical Sciences, The University of Tokyo in 2005 (Professor Tomohiko Ohwada). After working as a postdoctoral fellow in RIKEN in the laboratory of Professor Mikiko Sodeoka in 2005-2006 and Columbia University in the laboratory of Professor Koji Nakanishi in 2006-2007, she became an assistant professor of Professor Ohwada lab. She has been working in the lab and has been an associate professor since 2019. Her research interest is synthesis of unnatural amino acids and conformation analysis of oligopeptides. 